\title{
Hotspot KRAS mutations in brain metastases at the first metastatic recurrence of cutaneous melanoma
}

Roy Rabbie*1,2 , Peter Ferguson* ${ }^{3,4,5}$, Kim Wong ${ }^{1}$, Una Moran ${ }^{7}$, Clinton Turner ${ }^{6}$, Patrick Emanuel $^{6}$, Kerstin Haas ${ }^{10}$, Jodi M. Saunus ${ }^{8,9}$, Morgan R. Davidson ${ }^{8,9}$, Sunil R. Lakhani ${ }^{8,9}$, Brindha Shivalingam ${ }^{3}$, Georgina V. Long ${ }^{3,4,11}$, Christine Parkinson ${ }^{2}$, Iman Osman ${ }^{7}$, Richard A. Scolyer ${ }^{3,4,5}$, Pippa Corrie ${ }^{2}$, David J. Adams ${ }^{\S, 1}$

1. Experimental Cancer Genetics, The Wellcome Sanger Institute, Hinxton, Cambridgeshire, UK

2. Cambridge Cancer Centre, Cambridge University Hospitals NHS Foundation Trust, Cambridge, UK

3. Melanoma Institute Australia, The University of Sydney, Sydney, NSW, 2065, Australia.

4. Sydney Medical School, The University of Sydney, Sydney, NSW, 2006, Australia

5. Royal Prince Alfred Hospital, Camperdown, NSW, 2050, Australia

6. The University of Auckland, New Zealand

7. Interdisciplinary Melanoma Program, New York University School of Medicine, NY, USA

8. The University of Queensland Faculty of Medicine, UQ Centre for Clinical Research, Herston, Queensland, Australia

9. Pathology Queensland, Royal Brisbane Women's Hospital, Herston, Queensland, Australia

10. Francis Crick Institute, London, UK

11. Royal North Shore and Mater Hospitals, Sydney, NSW, 2065, Australia

*These authors contributed equally to this work

${ }^{\S}$ Correspondence to:

Dr. David Adams

Experimental Cancer Genetics

Wellcome Sanger Institute

Hinxton, Cambridge, CB10 1HH

Ph: +44 122383496862

Email: da1@sanger.ac.uk 


\section{ABSTRACT}

IMPORTANCE: Brain metastases occur in $60 \%$ of patients with advanced melanoma and are a major cause of melanoma-related mortality and morbidity. Although our understanding of the molecular alterations associated with melanoma progression is improving, there are currently no validated biomarkers which might help identify those patients at highest risk of developing brain metastases.

OBJECTIVE: To examine the somatic mutational and copy-number landscape of brain metastases that develop as the isolated first visceral site of recurrence - "early brainmetastasis" compared to extracranial melanoma metastases.

DESIGN, SETTING AND PARTICIPANTS: Whole-exome sequencing of 50 tumors from patients undergoing surgical resection of one or more brain metastasis occurring as the first site of visceral relapse were identified from prospectively maintained databases in Sydney, Wellington, New York and Cambridge. Whole exome sequencing analyses allowed mutational profiles to be compared to cutaneous melanomas in The Cancer Genome Atlas (SKCM-TCGA; $\mathrm{n}=358$ ) and the Memorial Sloan Kettering (SKCM-MSK-IMPACT; $\mathrm{n}=186$ ) datasets. An external dataset comprising a further 18 patients with surgically resected early brain metastasis from two additional academic centers served as an independent validation cohort.

MAIN OUTCOMES AND MEASURES: To assess the frequency of driver mutations in early brain metastasis and their influence on survival.

RESULTS: In concordance with the landmark melanoma sequencing studies, we identified mutations in $\operatorname{BRAF}(21 / 50,42 \%), \operatorname{NRAS}(14 / 50,28 \%)$ and $N F 1(11 / 50,22 \%)$ as the most frequently mutated melanoma driver genes. When compared to the mutational landscape of cutaneous melanomas in TCGA (SKCM-TCGA), KRAS was the most significantly enriched driver gene, with $5 / 50(10 \%)$ of brain metastases harboring non-synonymous mutations, of which 4/5 (80\%) were in the hotspot positions of codons 12 and 61 . This was significantly higher than the corresponding frequency of KRAS-mutations within the entire SKCM-TCGA (2\% (7/358), $p=0.009$, Fisher's Exact Test) as well as the SKCM-MSK-IMPACT cohort $(1.6 \%(3 / 186), p=0.016)$. 
Variants in KRAS were mutually exclusive from BRAF ${ }^{V 600}$, NRAS and HRAS mutations and were associated with a significantly reduced overall survival from resection of brain metastasis (relative to KRAS-wild type brain metastases) in multivariate Cox proportional hazard models (HR 1.80, 95\% Cl 1.46-24.89, $\mathrm{p}=0.013$ ). Mutations in KRAS were also clonal and concordant with extracranial disease, which suggests these mutations are present within the primary tumor

CONCLUSIONS AND RELEVANCE: Our analysis, the largest to date, suggests that early metastases to the brain (presenting as the first site of visceral relapse) are characterized by significant enrichment of hotspot KRAS mutations, potentially implicating constitutive RASdriven cellular programs in neurotropic metastatic behavior in these cases. Based on these data, we suggest that screening for KRAS mutations might help identify those patients with primary melanoma at higher risk of brain metastases or poor survival, and could help inform future surveillance strategies. 


\section{Key Points}

Question What is the frequency of driver mutations in early melanoma brain metastases?

Findings In this study of 50 patients with melanoma metastasizing first to the brain, KRAS mutations were the most significantly enriched driver gene ( $n=5,10 \%$ of patients) when compared to landmark cutaneous melanoma studies. The high KRAS mutation frequency was also observed in an external validation cohort of 18 patients with early brain metastases. Mutations in KRAS were mutually exclusive from mutations in the key RAS signaling genes and conferred a worse overall survival from resection of brain metastasis.

Meaning Hotspot KRAS mutations could help identify those patients with primary melanoma at higher risk of brain metastases that may benefit from more intensive, protracted surveillance as well as earlier use of adjuvant therapy. 


\section{Background}

Metastases to the central nervous system (CNS) are observed in $~ 60 \%$ of cutaneous melanoma patients developing disseminated disease and up to $90 \%$ at autopsy. ${ }^{1}$ Early detection of intracerebral recurrences remains critical, as isolated or oligometastatic brain metastases may be more amenable to potentially curative locoregional therapies and immunotherapies have demonstrated greatest efficacy in patients with small, asymptomatic metastases. ${ }^{1-3}$ Early predictors of brain metastases could therefore help identify those patients most likely to benefit from closer surveillance of the brain as well as early use of adjuvant therapies.

Importantly, epidemiological data suggest that patterns of metastatic dissemination may be partially determined by the clinical characteristics of the primary tumor (e.g. scalp/high mitotic rate primary melanomas might have higher risk of brain metastases ${ }^{4}$ ). However, melanomas have considerable molecular diversity and several lines of evidence suggest that metastatic melanomas (including brain metastases) are genetically evolved from their primary tumor predecessors. $^{5}$

Interestingly, $15-20 \%$ of brain metastases present as the isolated first visceral site of recurrence. ${ }^{6}$ Primary tumors in these 'early brain metastasis' cases reported as thinner and of lower American Joint Committee of Cancer (AJCC) Stage when compared to other visceral metastases, challenging the current understanding of brain metastases as the final stage of tumor progression and suggesting these tumors could harbor distinct biological properties favoring early hematogenous dissemination to the brain. ${ }^{6}$ Our analyses of the mutational landscape of early brain metastasis highlights key molecular features that could inform future prognostic, surveillance and intervention strategies. 


\section{Methods}

\section{Study population}

Patients with available archival paraffin embedded melanoma brain metastases (in the absence of other sites of visceral disease, confirmed by CT or MRI imaging prior to neurosurgery) were selected from prospectively maintained databases at The Melanoma Institute of Australia $(n=34)$, The Wellington School of Medicine $(n=8)$, New York University School of Medicine $(n=4)$ and Cambridge University Hospitals $(n=4)$ (discovery cohort). Samples from patients selected from The University of Auckland and The University of Queensland Australia ( $n=18$ total) made up the external validation cohort. All neuro-resections were undertaken between 2008 and 2018 at the respective academic neurosurgical centers as part of routine clinical care. All cases were ethically approved by the local Institutional Review Boards, as well as by the Sanger Institute's human materials and data management committee. All samples and clinical details are listed in Supplementary Table 1.

The clinical and mutation data from The Cancer Genome Atlas (SKCM-TCGA) ${ }^{7}$, was downloaded from the cBioPortal. The SKCM-MSK-IMPACT dataset was extracted from the publication by Zehir et $a l^{8}$ (Supplementary Methods).

\section{DNA sequencing}

Exome capture of the discovery cohort was performed using Agilent SureSelect All Exon V5 bait. Paired-end sequencing was performed using the Illumina HiSeq (Illumina, San Diego, CA, USA) platform at the Wellcome Sanger Institute. In $(n=16)$ cases where no matching germline DNA was available, a panel of 39 FFPE-extracted normals were used to filter germline variants as well as artefacts. MuTect (v1.1.7) and Sequenza (v2.1.2) were used to call somatic SNVs and copy number aberrations, respectively. Panel sequencing of the 18 external validation cohort samples was performed using custom pull-down sequencing (Supplementary Methods). 


\section{Survival analyses}

Univariate Kaplan-Meier survival plots were constructed using the $R$ 'Survival' package version 2.4.2. Univariate and multivariate hazard ratios (HR), $95 \%$ confidence intervals $(95 \% \mathrm{Cl})$ and corresponding p-values were obtained using Cox proportional hazards regression models. Multivariate corrections were undertaken for gender, age, as well as BRAF and NRAS mutation status. 


\section{Results}

Fifty patients who developed brain metastases as their first site of visceral disease spread were enrolled as part of the discovery cohort and were represented by a relatively high proportion of thin (T1-T2) $(n=25,50 \%)$ and non-ulcerated $(n=26,52 \%)$ primary melanomas (Supplementary Table 1).

Mutations in BRAF were detected in 21 (42\%) tumors, of which 15/21 (71\%) were in the V600 hotspot (Figures 1A \& 1B). NRAS mutations were identified in $14(28 \%)$ tumors and were all in hotspot positions on exons 2 (codons 12 and 13) and 3 (codon 61) and mutually exclusive from $B R A F^{\mathrm{V} 600}$ mutations. Comparing the mutational landscape of brain metastases to that of cutaneous melanomas in the SKCM-TCGA dataset (see Supplementary Methods), KRAS was the most significantly enrichment driver gene in our dataset, mutated in $10 \%$ (5/50) vs $2 \%(7 / 358)$ in the entire SCKM-TCGA collection, $p=0.009$ two-tailed Fisher's exact test). The mutation frequency of KRAS was also significantly enriched relative to the frequency of extracranial melanoma metastases, $10 \%(5 / 50)$ in our dataset vs $2.1 \%(6 / 274)$ in extracranial melanoma metastases in SKCM-TCGA ( $p=0.016$, two-tailed Fisher's exact test, see Supplementary Methods). Further, only $1.6 \%$ (3/186) of melanoma cases in the SKCM-MSK-IMPACT dataset ${ }^{8}$ were KRAS mutant, significantly lower than in our early brain metastasis discovery cohort ( $p=0.016$ two-tailed Fisher's exact test). Mutations in KRAS had a high variant allele frequency, indicating that these likely represent clonal (early) driver mutations (Table 1). Of note, three extracranial metastases available for sequencing from patients with KRAS-mutant brain metastases also harbored the same brain-metastatic KRAS mutations, suggesting that KRAS mutations were concordant with extracranial metastases (see Supplementary Methods). Notably, four out of five of the brain metastases mutations in KRAS were in hotspot codons 12 and 61 and mutually exclusive from other mutations in the mitogen-activated protein kinase (MAPK) signaling genes including $B R A F^{V 600}, N R A S$ and HRAS (Figure 1B). 
We conducted a further custom pull-down validation experiment on selected melanoma driver mutations within the discovery cohort and confirmed 56/60 (93\%) to be somatic mutations (see Methods). We also conducted another external validation experiment, analyzing a further 18 early metastases independently acquired from two different neurosurgical centers (see Supplementary Methods). This revealed 1 brain metastasis (5.6\%) harbored a $K R A S^{G 13 C}$ mutation, which was also mutually exclusive from mutations in the RAS signaling genes (BRAF/NRAS/HRAS) (Supplementary Figure 1). The copy number landscape of the early brain metastasis discovery cohort proved remarkably similar to that of SKCM-TCGA (Figure 1C \& Supplementary Figure 2).

All patients with KRAS-mutant brain metastases succumbed to disease, with a median overall survival from resection of brain metastasis of only 3 months, compared to 12 months in patients with resected KRAS-wild type brain metastases (HR 1.80, 95\% Cl 1.46-24.89, p=0.013, covariate corrected Cox proportional hazards model, Figures 2A \& B). Melanoma patients with tumors harboring KRAS mutations or amplifications represented in the SCKM-TCGA dataset were also associated with worse overall survival compared to KRAS wild-type melanomas (HR 2.6, $95 \% \mathrm{Cl} 1.2-5.4, \mathrm{p}=0.015$ univariate Cox-regression), although this did not meet the threshold for statistical significance after correction of clinical covariates, likely due to the limited sample size (HR 2.1, 95\% Cl 0.9-4.7, $\mathrm{p}=0.07$, multivariate corrected Cox proportional hazards regression, Supplementary Figure 4). 


\section{Discussion}

This analysis represents the largest survey of mutation profiles of melanoma brain metastases and the first to show an association between KRAS mutations and adverse outcomes in melanoma. The predominance of KRAS mutations in codons 12,13 and 61 , as well as the mutual exclusivity to other key drivers of MAPK signaling suggests these likely represent important drivers in this context.

The RAS family of GTPases consists of genes including NRAS, KRAS and HRAS, mutated in $25 \%$, $2 \%$ and $1 \%$ of melanomas respectively. ${ }^{7}$ NRAS-mutant melanomas are recognized to be more aggressive and associated with poorer outcomes, however very little is known about KRASmutant melanoma. ${ }^{9}$ KRAS-mutant early brain metastasis in our study generally emanated from thin and non-ulcerated primary melanomas (Table 1). Hence, KRAS detection might in future be used to 'upstage' a subgroup of lower risk patients not currently offered routine surveillance and/or adjuvant therapy potentially avoiding the devastating impact of brain metastases. Mutations in KRAS were clonal and concordant with extracranial disease, which suggests these mutations are present within the primary tumor, however further studies will be required to confirm this.

The MAPK and phosphoinositide-3 kinase (PI3K) pathways are the two key downstream signaling pathways through which constitutively activated RAS exerts its pro-tumorigenic effects. MAPK pathway activation and brain metastases are inextricably connected and BRAF and NRAS mutations are associated with an increased risk of brain metastasis. ${ }^{10}$ In the same way the PI3K/AKT pathway has been mechanistically linked with the development of brain metastases $^{11}$, and analyses of patient-matched pairs of brain and extracranial metastases have revealed that brain metastases have higher levels of activated AKT and lower expression of PTEN, a finding also observed using immunohistochemistry. ${ }^{12}$ Hotpot KRAS mutations are known to activate EGFR signaling pathways, which in-turn is associated with an increased risk of brain metastases in non-small cell lung cancer $(\mathrm{NSCLC})^{13}$. One human sequencing study has further shown a direct association between KRAS-mutations and NSCLC brain metastases ${ }^{14}$, although further functional validation will be required. 


\section{Limitations}

The retrospective nature of this analysis could feasibly introduce a degree of selection bias, in particular by only identifying those patients with operable early brain metastasis we might have excluded a larger patient demographic with more widespread disease. Emerging evidence indicates that metastatic outgrowth may also depend on the interplay between cancer cells and the host stroma ${ }^{15}$ however such tumor-cell extrinsic factors might not be fully captured by this analysis. The identification of KRAS mutations as a predictive biomarker for the development of early brain metastases will ultimately require prospective validation in larger cohorts employing multivariate models, particularly assessing its predictive value in relation to other clinical covariates ${ }^{4}$.

\section{Conclusion}

In summary, our analyses indicate that the patterns of melanoma recurrence may be at least partially determined by the tumor mutational profile, and that up to $10 \%$ of patients developing early brain metastases may have tumors driven by oncogenic KRAS mutations. This observation has implications for deciphering the biology of site-specific metastatic pathogenesis and, if validated in larger prospectively curated cohorts, might influence prognosis, surveillance and intervention in patients carrying these somatic alterations. 
Acknowledgements: First and foremost, we would like to sincerely thank the patients involved in this study. We would also like to thank the Cancer, Ageing and Somatic Mutation Program team at the Sanger Institute for facilitating QC and DNA sequencing, particularly Claire Hardy, Stephen Gamble and Elizabeth Anderson. We would also like to thank Ingrid Ferreira for performing the histopathological analyses for the University of Queensland cases. Sofia Chen, James Gilbert and Matthew Garnett for their help with the custom capture bait. Assistance from colleagues at Melanoma Institute Australia and Royal Prince Alfred Hospital is gratefully appreciated. Carla Daniela Robles Espinoza for critical review of the data and the manuscript. 


\section{References}

1. Ajithkumar, T., Parkinson, C., Fife, K., Corrie, P. \& Jefferies, S. Evolving treatment options for melanoma brain metastases. Lancet Oncol 16, e486-97 (2015).

2. Tawbi, H.A. et al. Combined Nivolumab and Ipilimumab in Melanoma Metastatic to the Brain. N Engl J Med 379, 722-730 (2018).

3. Long, G.V. et al. Combination nivolumab and ipilimumab or nivolumab alone in melanoma brain metastases: a multicentre randomised phase 2 study. Lancet Oncol 19, 672-681 (2018).

4. Haydu, L.E. et al. Cumulative Incidence and Predictors of CNS Metastasis for Patients With American Joint Committee on Cancer 8th Edition Stage III Melanoma. J Clin Oncol, Jco1901508 (2020).

5. Dagogo-Jack, I., Carter, S.L. \& Brastianos, P.K. Brain Metastasis: Clinical Implications of Branched Evolution. Trends Cancer 2, 332-337 (2016).

6. Ma, M.W. et al. Challenging the current paradigm of melanoma progression: brain metastasis as isolated first visceral site. Neuro Oncol 14, 849-58 (2012).

7. Genomic Classification of Cutaneous Melanoma. Cell 161, 1681-96 (2015).

8. Zehir, A. et al. Mutational landscape of metastatic cancer revealed from prospective clinical sequencing of 10,000 patients. Nat Med 23, 703-713 (2017).

9. Jakob, J.A. et al. NRAS mutation status is an independent prognostic factor in metastatic melanoma. Cancer 118, 4014-23 (2012).

10. Adler, N.R. et al. Tumour mutation status and sites of metastasis in patients with cutaneous melanoma. Br J Cancer 117, 1026-1035 (2017).

11. Zhang, L. et al. Microenvironment-induced PTEN loss by exosomal microRNA primes brain metastasis outgrowth. Nature 527, 100-104 (2015).

12. Chen, G. et al. Molecular profiling of patient-matched brain and extracranial melanoma metastases implicates the PI3K pathway as a therapeutic target. Clin Cancer Res 20, 5537-46 (2014).

13. Tan, L. et al. A Comprehensive Meta-Analysis of Association between EGFR Mutation Status and Brain Metastases in NSCLC. Pathol Oncol Res 25, 791-799 (2019). 
14. Zhao, N. et al. Alterations of LKB1 and KRAS and risk of brain metastasis: comprehensive characterization by mutation analysis, copy number, and gene expression in non-smallcell lung carcinoma. Lung Cancer 86, 255-61 (2014).

15. Quail, D.F. \& Joyce, J.A. The Microenvironmental Landscape of Brain Tumors. Cancer cell 31, 326-341 (2017). 


\section{Figure legends}

Figure 1. Tile plot of melanoma driver mutations in the early brain metastasis discovery cohort $(n=50)$. A) The mutational profiles of brain metastases are indicated. Mutational load was calculated as the number of non-synonymous mutations per $\mathrm{Mb}$, median is indicated by the solid horizontal grey line and the $95 \%$ confidence interval by the dashed lines (median 38 mutations/Mb, 95\% Cl 14.2-67.1). The genes shown carry non-synonymous mutations within the selected melanoma drivers outlined in Hayward et al. $(n=19)$ and are ordered according to their mutation frequency within this cohort. The corresponding mutational load and gene mutational frequencies in the SKCM-TCGA dataset $(n=358)$ is indicated in the plot aside. SKCMTCGA mutational load boxplots are colored according to whether the sample was classified as a primary ( $n=75$, blue) or metastasis ( $n=283$, red). The median non-synonymous mutational load in SKCM-TCGA is indicated by the solid horizontal grey line and the $95 \%$ confidence interval by the dashed lines (median 9.9 mutations/Mb, 95\% Cl 4.4-18.3). *signifies the gene is significantly more mutated in the early brain metastasis cohort. KRAS; $10 \%(5 / 50)$ vs $2 \%(7 / 358)$ in the SCKM-TCGA collection, $\mathrm{p}=0.009$ Fisher's exact test), $R B 1 ;(6 / 5012 \%$ in our dataset vs $3.9 \%$ 14/358 in SKCM-TCGA, p=0.025 Fisher's exact test). B) Focused tile plot from (A), highlighting the mutated amino acid positions within the RAS signaling genes. As expected, mutations in NRAS were mutually exclusive to $B R A F^{V 600}$ hotspots. The four hotspot KRAS mutations were also mutually exclusive to $B R A F^{\mathrm{v} 600}$ and to mutations in NRAS and HRAS. Mutations shaded in grey were not found in the cBio catalogue of cancer mutations and likely represent passenger mutations. C) Copy number profile of the early melanoma metastasis discovery cohort $(n=30)$ overlaid onto the copy number profile of SKCM-TCGA $(n=337)$. The non-overlaid plots are shown in Supplementary Figure 2. 
Figure 2. Impact of KRAS mutational status on survival. A) Kaplan-Meier survival curves showing overall survival probabilities from resection of brain metastasis (defined as the time from the resection of the brain metastasis to last follow-up (censored) or death from any cause) for the hotspot KRAS-mutant $(\mathrm{n}=4)$ versus KRAS-wild-type $(\mathrm{n}=43)$ patients (7 KRAS wild-type patients did not have survival data available). Patients with KRAS-mutant tumors had significantly worse overall survival from resection of brain metastasis than KRAS-wild-type patients, median 3 vs 12 months ( $p=0.002$, univariate Cox regression). B) Forest plot comparing KRAS-mutant versus wild-type survival from resection of brain metastasis in univariate (HR 5.58, 95\% Cl 1.80-17.24, p=0.003) and multivariate (HR 6.02, 95\% Cl 1.46-24.89, p=0.013) Cox proportional hazards regression models. Multivariate correction was undertaken for gender, age at resection of brain metastasis, as well as BRAF and NRAS mutation status.

Table 1. Clinical and mutational characteristics of the patients with hotspot KRAS mutations (including both patients from the discovery and validation cohorts). Of note all hotspot KRAS mutations were identified in patients with either thin (T1/T2) or no prior history of primary melanoma, and from primary tumors in chronically sun-exposed locations. Mutations in KRAS also had a high variant allele frequency, indicating that these likely represent clonal driver mutations which is further supported by their concordance in extracranial metastases. Cambridge: Cambridge University Hospitals, MIA: Melanoma Institute of Australia, Queensland: The University of Queensland Australia. SSM: Superficial spreading melanoma, NM: Nodular melanoma, LMM: Lentigo-maligna melanoma.

Supplementary Figure 1. Tile plot of mutated positions within the RAS signaling genes in the external validation cohort $(\mathbf{n}=\mathbf{1 8})$. The $K R A S^{G 13 C}$ mutation was mutually exclusive from both $B R A F$ and NRAS hotspot mutations, in keeping with the findings from the discovery cohort (Figure 1B).

Supplementary Figure 2. Copy number profile of early melanoma brain metastasis from the discovery cohort (top, $n=30$ ) and the SKCM-TCGA (bottom, $n=337$ ).

Supplementary Figure 3. Kaplan-Meier survival curves of the early brain metastasis discovery cohort. A) Overall survival from primary disease (defined as the time from the resection of the 
primary tumor to last follow-up (censored) or death from any cause). Shaded regions represent the $95 \%$ confidence interval (median survival from primary disease 65.0 months, $95 \% \mathrm{Cl} 45.0$ 125.0 months). Survival data from primary disease was only available on 42 patients ( 3 patients had no history of primary melanoma, 5 patients had no survival information). B) Overall survival from resection of brain metastasis, defined as the time from the resection of brain metastasis to last follow-up (censored) or death from any cause. Median 12.0 months, $95 \% \mathrm{Cl}$ 6.0-62.0 months. Data from resection of brain metastasis was available on 47 patients. C) Impact of KRAS mutational status on overall survival from primary tumor. D) Forest plot comparing KRASmutant versus KRAS-wild-type overall survival from primary tumor in univariate (HR 2.74, 95\% $\mathrm{Cl}$ 0.92- 8.21, $\mathrm{p}=0.071$ ) and multivariate ( $\mathrm{HR}$ 8.46, 95\% $\mathrm{Cl}$ 0.34-207.61, $\mathrm{p}=0.191$ ) Cox proportional hazards regression models. Multivariate correction was undertaken for gender, age at primary tumor resection, T-stage and ulceration of primary tumor, as well as BRAF and NRAS mutation status.

Supplementary Figure 4. Impact of KRAS mutational status on overall survival in SKCM-TCGA. A) Kaplan-Meier curves for overall survival in KRAS hotpot mutations or amplifications $(n=10)$ versus KRAS-WT ( $\mathrm{n}=341$ ) melanoma in SKCM-TCGA. B) Overall survival in KRAS hotpot mutation only $(n=6)$ versus KRAS-WT ( $n=345)$ melanoma in SKCM-TCGA. C) Forest plot showing KRASmutant survival in SKCM-TCGA (either hotspot mutations or amplifications $n=10$, or hotpot mutations alone $\mathrm{n}=6)$ in univariate ( $\mathrm{HR}$ 2.59, 95\% Cl 1.21- 5.55, $\mathrm{p}=0.015$ for mutation/amplification and $H R$ 1.37, $95 \% \mathrm{Cl} 0.44-4.31, p=0.587$ for mutation alone) and multivariate (HR 2.10, 95\% Cl 0.94-4.67, p=0.070 for mutation/amplification and $H R$ 1.37, 95\% $\mathrm{Cl}$ 0.41-4.53, $\mathrm{p}=0.605$ for mutation alone) Cox proportional hazards regression models. Multivariate correction was undertaken for stage, sex, age at diagnosis of primary, nonsynonymous mutation count as well for BRAF and NRAS mutation status.

Supplementary Table 1. Demographic and clinical characteristics of all samples in the discovery cohort $(\mathbf{n}=\mathbf{5 0})$. Germline and sequenced extracranial samples (the latter with concordant KRAS mutations) are also indicated in column "Tissue type".

Supplementary Table 2. Clinical characteristics of hotspot KRAS-mutant ( $n=4)$ vs KRAS-wild type $(n=46)$ patient within the discovery cohort. 
Supplementary Table 3. Clinical and mutational characteristics of the patients with hotspot KRAS mutations in SKCM-TCGA ( $n=6)$ and SKCM-MSK-IMPACT ( $n=2)$. KRAS mutations were mutually exclusive to BRAF/NRAS/HRAS hotspot mutations in both datasets.

Supplementary Table 4. List of cancer driver genes $(n=549)$ included in the custom capture bait used on the $(n=18)$ external validation samples.

Supplementary Table 5. List of cancer driver genes $(n=278)$ included in the custom capture bait used on the orthogonal validation of driver SNVs $(n=60)$ from the discovery cohort. 
Funding/support: This work was supported by Cancer Research UK and the Wellcome Trust. NHMRC Program Funding to SRL (APP1113867). NHMRC Program Funding to RAS and GVL (APP1093017). RAS and GVL are supported by NHMRC Practitioner Fellowships. GVL is supported by the University of Sydney Medical Foundation.

Role of the funder/sponsor: The funders/sponsors had no role in the design and conduct of the study; collection, management, analysis and interpretation of the data, preparation, review or approval of the manuscript and decision to submit the manuscript for publication.

Author contributions: R.R. coordinated the clinical and molecular data extraction, sequencing, analyzed the data and wrote the paper; P.F. coordinated the samples and clinical data extraction from both the MIA and centers across New Zealand and co-wrote the paper; K.W. performed the bioinformatic analyses including somatic variant and copy number calling and plotted the copy number profiles. C.T. and P.E. identified the appropriate cases from across New Zealand, and extracted all the samples and associated clinical data. U.M. identified the appropriate cases from New York University Medical Center and extracted all the samples and associated clinical data. J.M.S. and M.R.D. identified the appropriate cases from the University of Queensland and extracted all the samples and associated clinical data. S.R.L. (The University of Queensland), I.O. (New York University Medical Center), G.V.L. and R.A.S. (The Melanoma Institute Australia) funded and facilitated provision of patient samples, patient's data, materials, reviewed manuscript, and provided senior input from the respective comprehensive cancer centers. I.O. was the first to describe 'brain metastases developing as the first site of visceral relapse' ${ }^{6}$ and provided expert input on this phenomenon. P.C. provided clinical supervision including critical review of the manuscript. D.J.A. provided overall supervision on all aspects of the study as well as critical review of the manuscript. All authors approved the final version.

\section{Conflicts of Interest}

G.V.L is consultant advisor to Amgen, Aduro, Array, BMS, MERCK, Novartis, Roche, Pierre-Fabre. R.A.S has received fees for professional services from MSD, BMS, Novartis, Myriad, NeraCare. 


\section{Fig 1}

sex IIIIIIIIIIIIIIIIIIIIIIIIIIIIIIIIIIIIIII

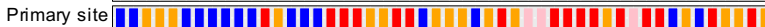
Histologic subtype

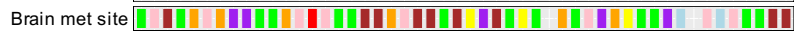

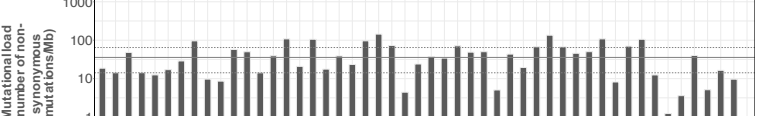
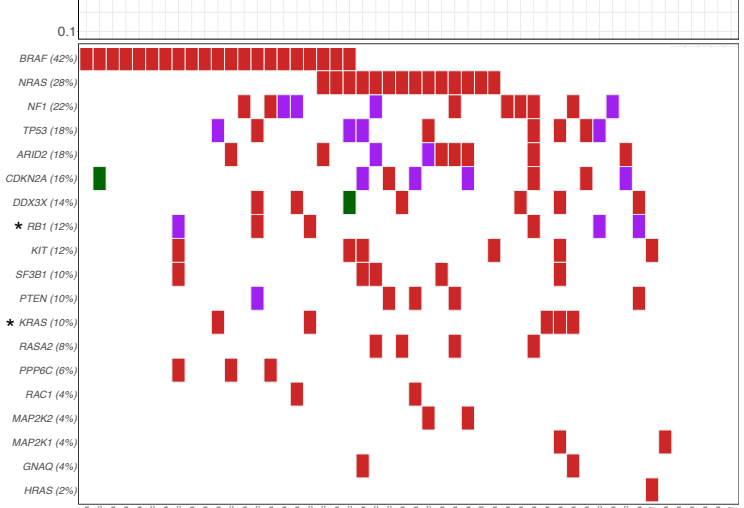

ICGA-SKCM $(n=358)$

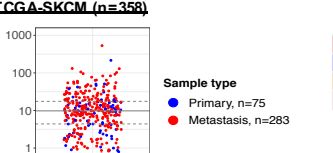

Histologic subtype

- Superficial spreading

Nodular

Melanoma-in-situ

Acral lentiginous

No known primary

Brain met site

- Frontal

- Occipital

Cerebellum

Intradural

Tromporal

Parieto-occipital

Parasagittal

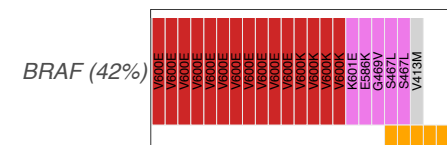

NRAS (28\%)
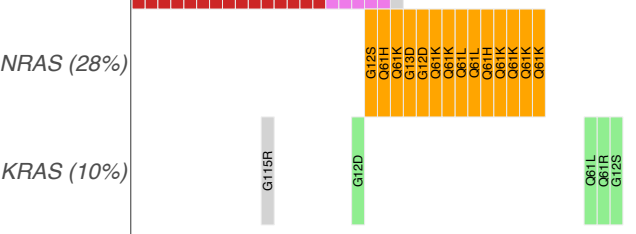

Amino acid position

- BRAFVGOO

nOM-B
NRAS
KRAS
HRAS

Not in COSMIC

HRAS (2\%)

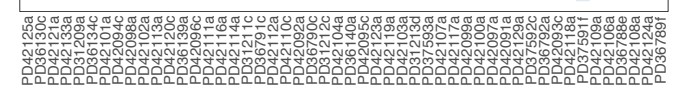

C

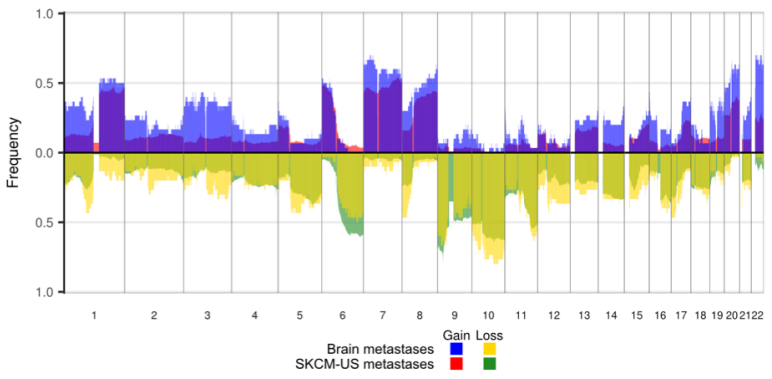

Figure 1. Tile plot of melanoma driver mutations in the early brain metastasis discovery cohort $(n=50)$. A) The mutational profiles of brain metastases are indicated. Mutational load was calculated as the number of nonsynonymous mutations per $\mathrm{Mb}$, median is indicated by the solid horizontal grey line and the $95 \%$ confidence interval by the dashed lines (median 38 mutations/Mb, 95\% Cl 14.2-67.1). The genes shown carry non-synonymous mutations within the selected melanoma drivers outlined in Hayward et al. $(n=19)$ and are ordered according to their mutation frequency within this cohort. The corresponding mutational load and gene mutational frequencies in the SKCM-TCGA dataset $(n=358)$ is indicated in the plot aside. SKCM-TCGA mutational load boxplots are colored according to whether the sample was classified as a primary ( $n=75$, blue) or metastasis ( $n=283$, red). The median non-synonymous mutational load in SKCM-TCGA is indicated by the solid horizontal grey line and the $95 \%$ confidence interval by the dashed lines (median 9.9 mutations/Mb, 95\% Cl 4.4-18.3). *signifies the gene is significantly more mutated in the early brain metastasis cohort. KRAS; $10 \%(5 / 50)$ vs $2 \%(7 / 358)$ in the SCKM-TCGA collection, $p=0.009$ Fisher's exact test), RB1; (6/50 12\% in our dataset vs 3.9\% 14/358 in SKCM-TCGA, $\mathrm{p}=0.025$ Fisher's exact test). B) Focused tile plot from (A), highlighting the mutated amino acid positions within the RAS signaling genes. As expected, mutations in NRAS were mutually exclusive to BRAFV600 hotspots. The four hotspot KRAS mutations were also mutually exclusive to $B R A F^{\mathrm{V} 600}$ and to mutations in NRAS and HRAS. Mutations shaded in grey were not found in the cBio catalogue of cancer mutations and likely represent passenger mutations. C) Copy number profile of the early melanoma metastasis discovery cohort $(n=30)$ overlaid onto the copy number profile of SKCM-TCGA $(n=337)$. The non-overlaid plots are shown in Supplementary Figure 2. 
A

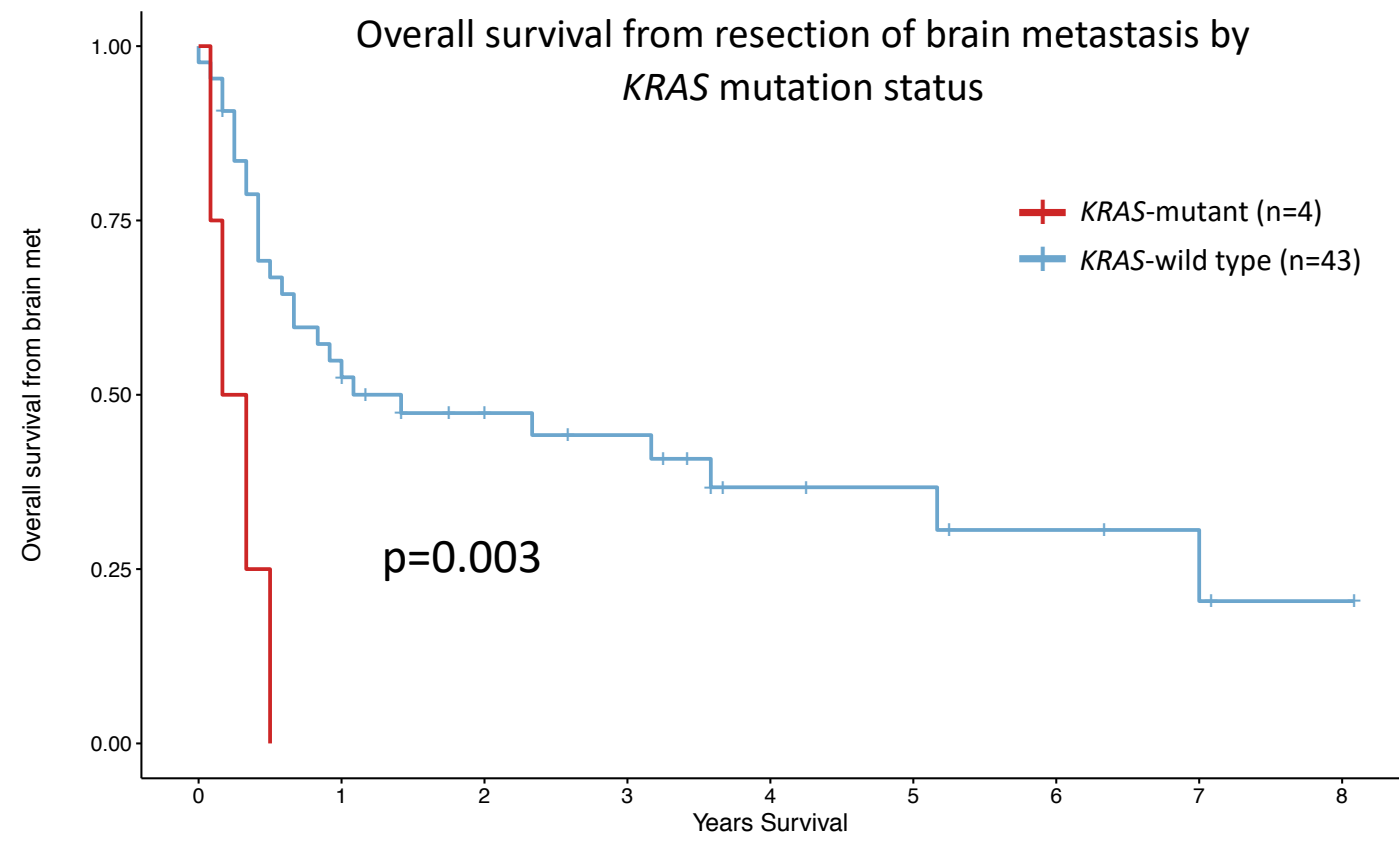

Number at risk

\begin{tabular}{|c|c|c|c|c|c|c|c|c|c|}
\hline KRAS-wild type & 43 & 23 & 16 & 13 & 7 & 6 & 4 & 3 & 1 \\
\hline \multirow[t]{2}{*}{ KRAS-mutant } & 4 & 0 & 0 & 0 & 0 & 0 & 0 & 0 & 0 \\
\hline & 0 & 1 & 2 & 3 & 4 & 5 & 6 & 7 & 8 \\
\hline
\end{tabular}

B

Forest plot, hotspot KRAS-mutations in survival from resection of brain-met

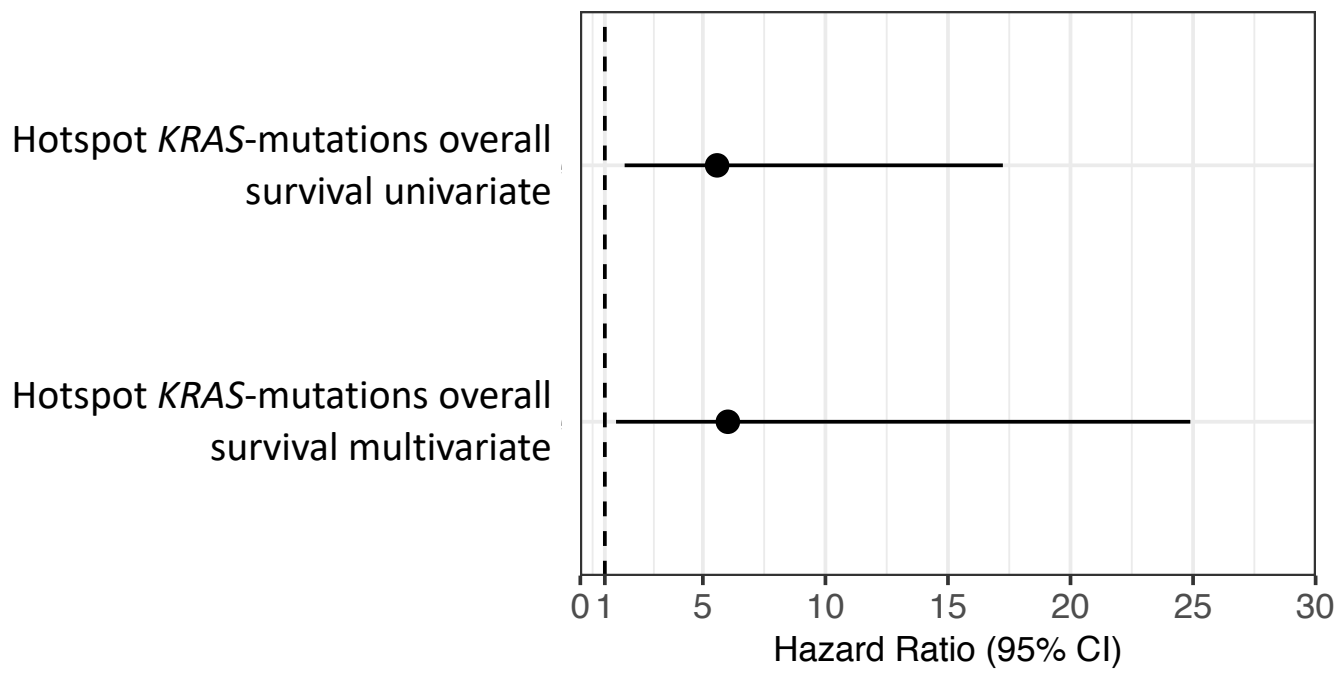

p-value

0.003

0.013

Figure 2. Impact of KRAS mutational status on survival. A) Kaplan-Meier survival curves showing overall survival probabilities from resection of brain metastasis (defined as the time from the resection of the brain metastasis to last follow-up (censored) or death from any cause) for the hotspot KRAS-mutant $(n=4)$ versus KRAS-wild-type $(n=43)$ patients ( 7 KRAS wild-type patients did not have survival data available). Patients with KRAS-mutant tumors had significantly worse overall survival from resection of brain metastasis than KRAS-wild-type patients, median 3 vs 12 months ( $p=0.002$, univariate Cox regression). B) Forest plot comparing KRAS-mutant versus wild-type survival from resection of brain metastasis in univariate (HR 5.58, 95\% Cl 1.80-17.24, $\mathrm{p}=0.003)$ and multivariate $(\mathrm{HR} 6.02,95 \% \mathrm{Cl}$ 1.46-24.89, $\mathrm{p}=0.013$ ) Cox proportional hazards regression models. Multivariate correction was undertaken for gender, age at resection of brain metastasis, as well as BRAF and NRAS mutation status. 


\section{Table 1}

\begin{tabular}{|c|c|c|c|c|c|c|c|c|c|c|c|c|}
\hline Patient_Pin & SampleID & Centre & Gender & Primary site & $\begin{array}{l}\text { Histologic } \\
\text { subtype }\end{array}$ & $\begin{array}{c}\text { Breslow } \\
\text { thickness } \\
(\mathrm{mm})\end{array}$ & $\begin{array}{c}\text { Ulcerati } \\
\text { on }\end{array}$ & $\begin{array}{l}\text { Clark } \\
\text { level }\end{array}$ & Mitoses & Tstage & $\begin{array}{c}\text { KRAS } \\
\text { mutated AA } \\
\text { position }\end{array}$ & $\begin{array}{l}\text { Variant } \\
\text { allele } \\
\text { frequency }\end{array}$ \\
\hline MBM_Disc_10 & PD42091a & MIA & Female & Extremity & SSM & 0.2 & No & II & 0 & $\mathrm{~T} 1$ & G12S & 0.85 \\
\hline MBM_Disc_23 & PD42097a & MIA & Male & Head/neck & NM & 1.5 & No & NA & NA & T2a & Q61R & 0.68 \\
\hline MBM_Disc_27 & PD42100a & MIA & Male & Head/neck & LMM & 1.5 & Yes & IV & 5 & $\mathrm{~T} 2 \mathrm{a}$ & Q61L & 0.86 \\
\hline MBM_Disc_40 & PD31211c & Cambridge & Male & Head/neck & $\begin{array}{c}\text { Melanoma- } \\
\text { in-situ }\end{array}$ & 0 & No & I & 0 & T1a & G12D & 0.50 \\
\hline MBM_Valid_10 & PD44959a & $\begin{array}{l}\text { University of } \\
\text { Queensland }\end{array}$ & Male & $\begin{array}{c}\text { No history } \\
\text { primary }\end{array}$ & $\begin{array}{c}\text { No history } \\
\text { primary }\end{array}$ & $\begin{array}{l}\text { No } \\
\text { history } \\
\text { primary }\end{array}$ & $\begin{array}{c}\text { No } \\
\text { history } \\
\text { primary }\end{array}$ & $\begin{array}{l}\text { No } \\
\text { history } \\
\text { primary }\end{array}$ & $\begin{array}{c}\text { No } \\
\text { history } \\
\text { primary }\end{array}$ & $\begin{array}{c}\text { No } \\
\text { history } \\
\text { primary }\end{array}$ & G13C & 0.70 \\
\hline
\end{tabular}

Table 1. Clinical and mutational characteristics of the patients with hotspot KRAS mutations (including both patients from the discovery and validation cohorts). Of note all hotspot KRAS mutations were identified in patients with either thin (T1/T2) or no prior history of primary melanoma and from primary tumors in chronically sun-exposed locations. Mutations in KRAS also had a high variant allele frequency, indicating that these likely represent clonal driver mutations. Cambridge: Cambridge University Hospitals, MIA: Melanoma Institute of Australia, Queensland: The University of Queensland Australia. SSM: Superficial spreading melanoma, NM: Nodular melanoma, LMM: Lentigo-maligna melanoma. 


\section{Supplementary Figure 2}

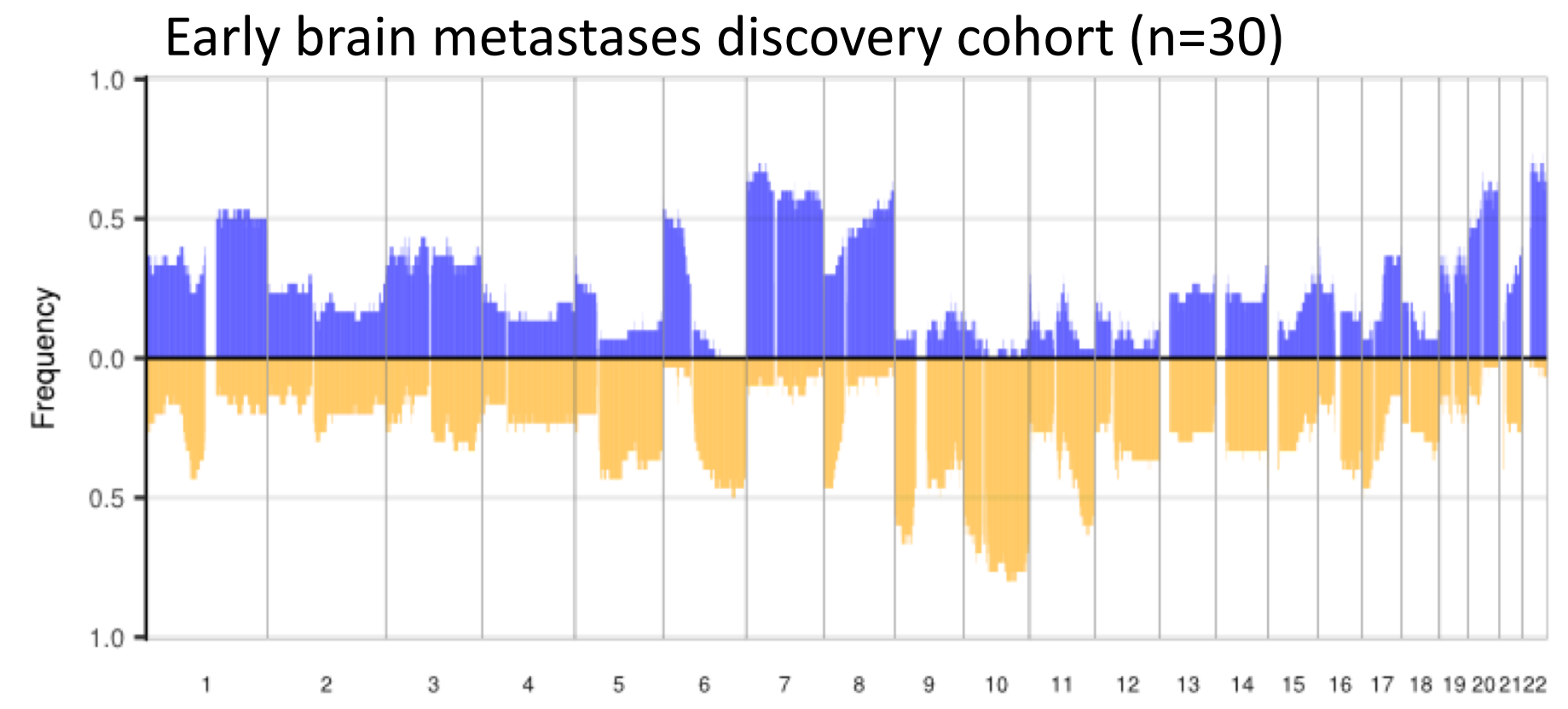

\section{SKCM-TCGA cohort $(n=337)$}

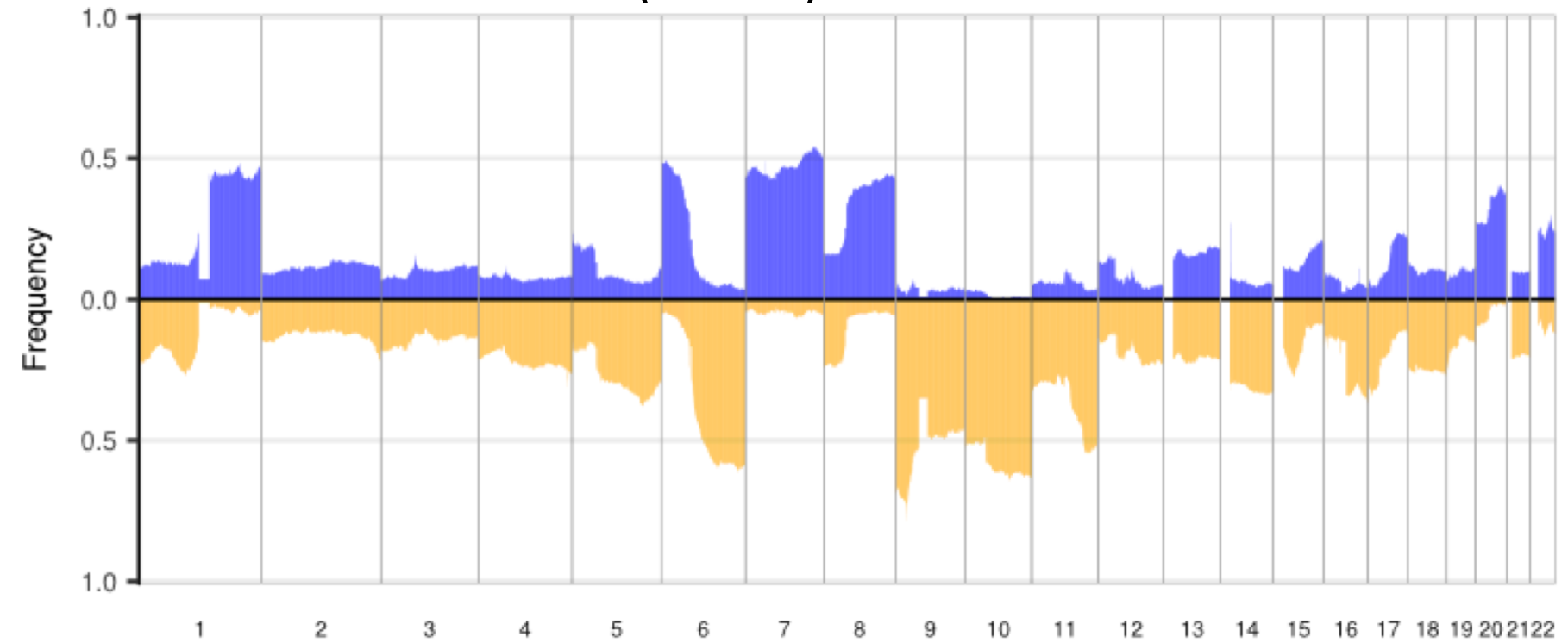

Supplementary Figure 2. Copy number profile of early melanoma brain metastasis from the discovery cohort (top, $\mathrm{n}=30$ ) and the SKCM-TCGA (bottom, $\mathrm{n}=337$ ). 


\section{Supplementary Figure 3}

A
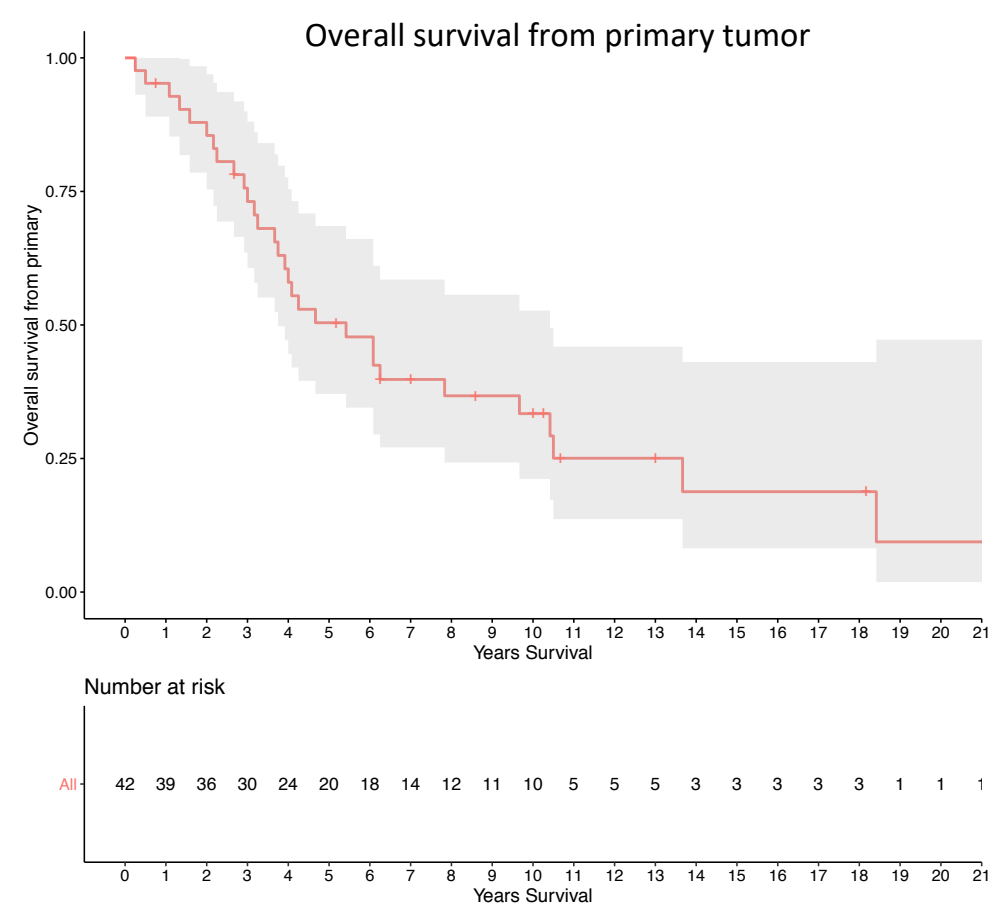

B

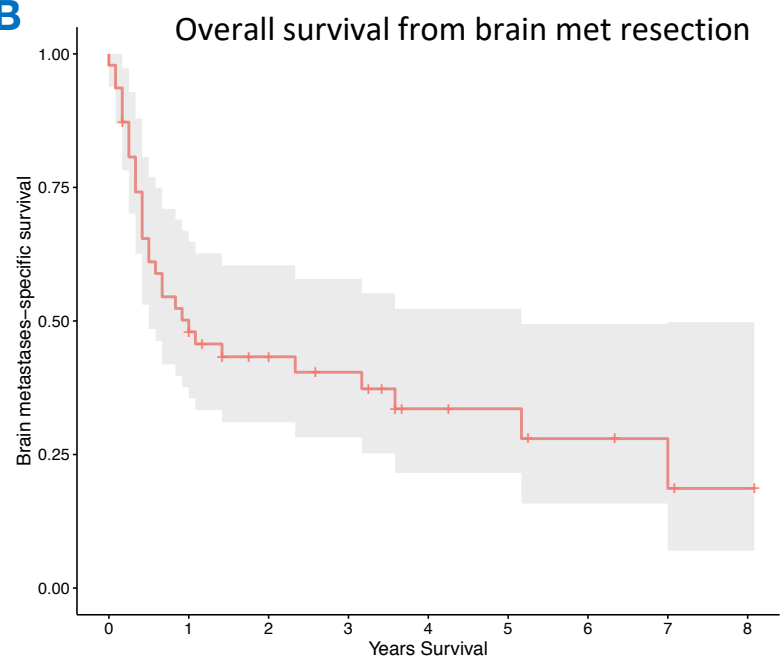

Number at risk

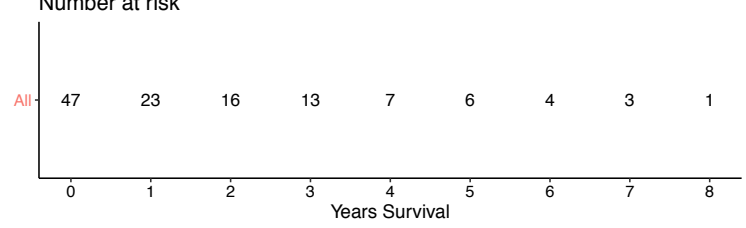

C

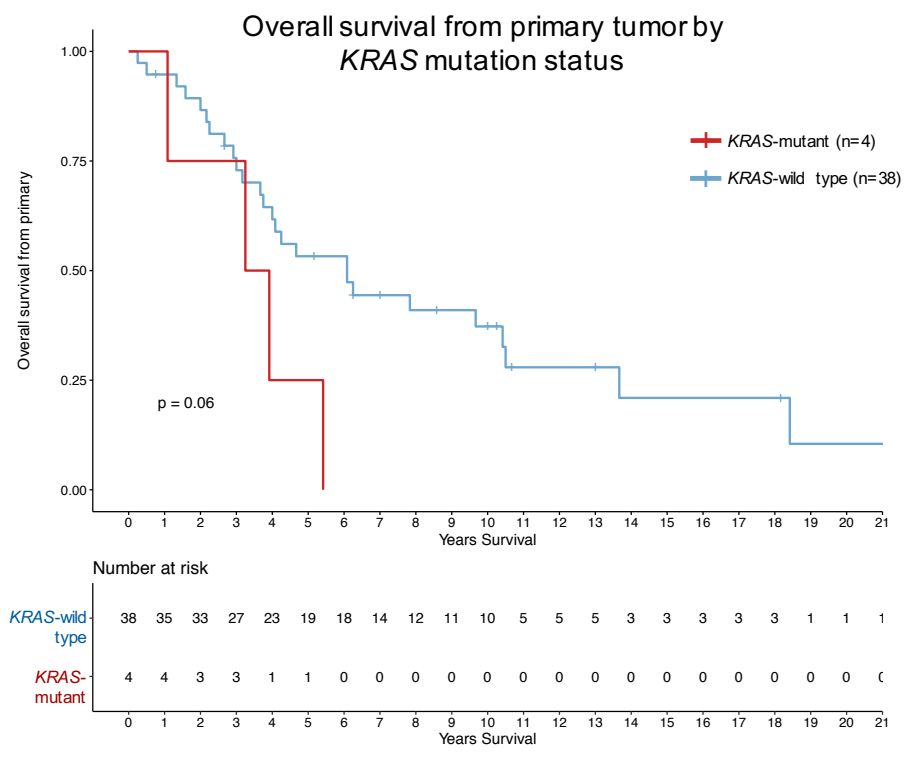

D

Forest plot, hotspot KRAS-mutations in overall survival from primary tumor

p-value

0.071

mutations univariate

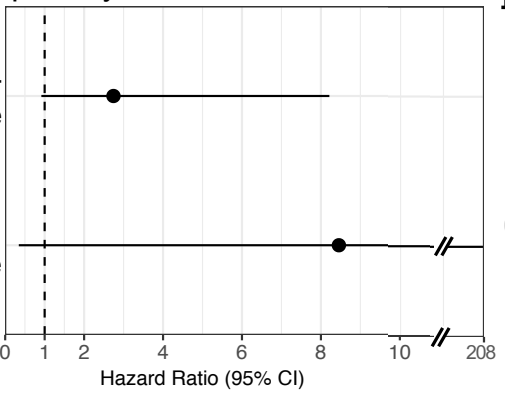

0.191

Supplementary Figure 3. Kaplan-Meier survival curves of the early brain metastasis discovery cohort. A) Overall survival from primary disease (defined as the time from the resection of the primary tumor to last follow-up (censored) or death from any cause). Shaded regions represent the $95 \%$ confidence interval (median survival from primary disease 65.0 months, $95 \% \mathrm{Cl}$ 45.0-125.0 months). Survival data from primary disease was only available on 42 patients ( 3 patients had no history of primary melanoma, 5 patients had no survival information). B) Overall survival from resection of brain metastasis, defined as the time from the resection of brain metastasis to last followup (censored) or death from any cause. Median 12.0 months, 95\% Cl 6.0-62.0 months. Data from resection of brain metastasis was available on 47 patients. C) Impact of KRAS mutational status on overall survival from primary tumor. D) Forest plot comparing KRAS-mutant versus KRAS-wild-type overall survival from primary tumor in univariate (HR 2.74, 95\% $\mathrm{Cl} 0.92-8.21, \mathrm{p}=0.071)$ and multivariate ( $\mathrm{HR} 8.46,95 \% \mathrm{Cl} 0.34-207.61, \mathrm{p}=0.191)$ Cox proportional hazards regression models. Multivariate correction was undertaken for gender, age at primary tumor resection, $\mathrm{T}$-stage and ulceration of primary tumor, as well as BRAF and NRAS mutation status. 


\section{Supplementary Figure 4}
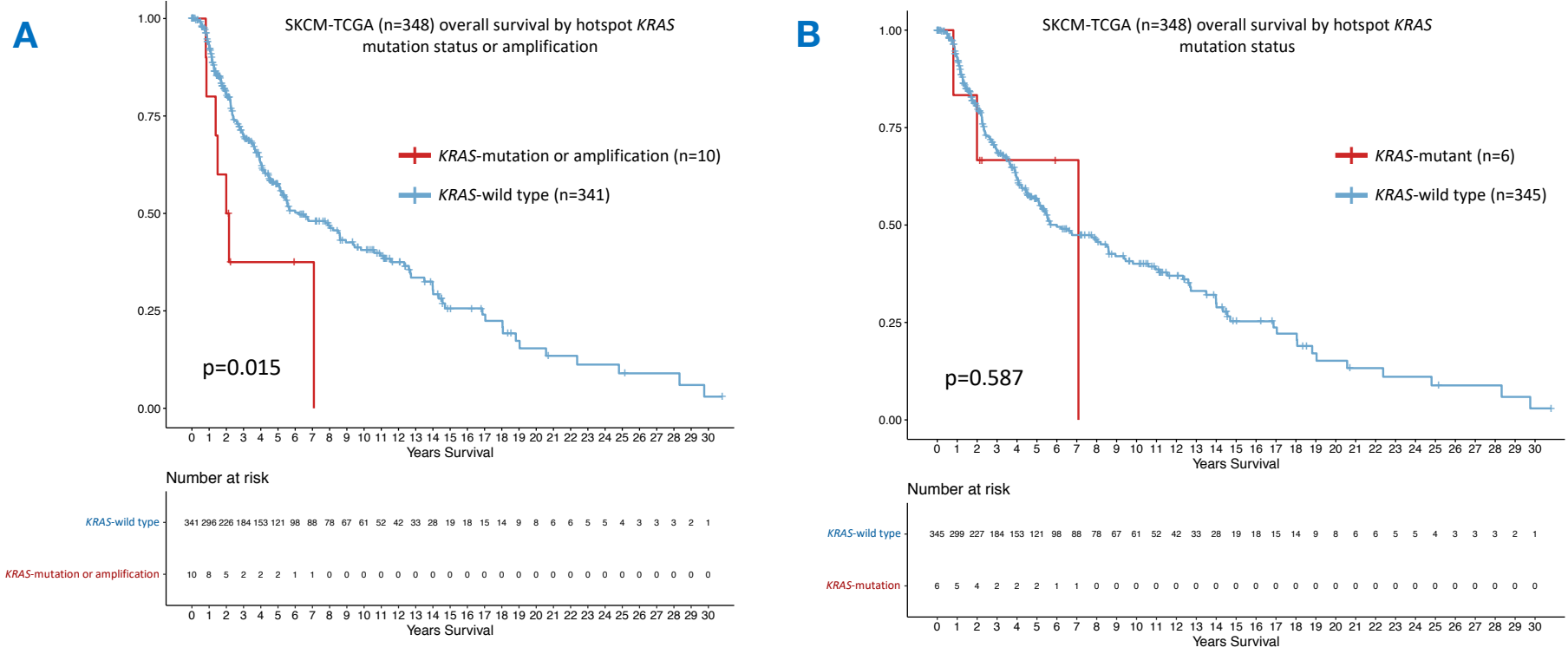

C

Forest plot, hotspot KRAS-mutations in SKCM-TCGA survival

p-value

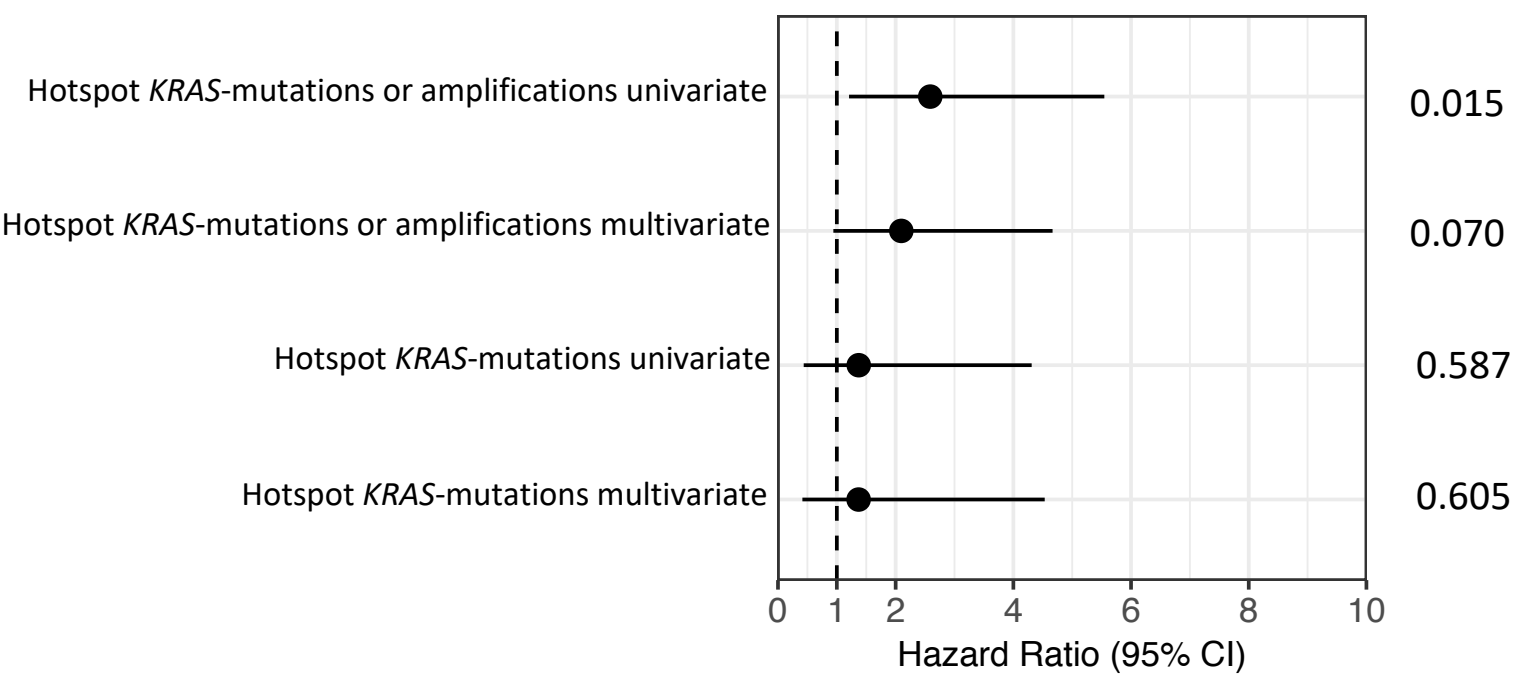

Supplementary Figure 4. Impact of KRAS mutational status on overall survival in SKCM-TCGA. A) Kaplan-Meier curves for overall survival in KRAS hotpot mutations or amplifications $(\mathrm{n}=10)$ versus $K R A S-$ WT $(n=341)$ melanoma in SKCM-TCGA. B) Overall survival in KRAS hotpot mutation only ( $n=6)$ versus KRAS-WT $(\mathrm{n}=345)$ melanoma in SKCM-TCGA. C) Forest plot showing KRAS-mutant survival in SKCM-TCGA (either hotspot mutations or amplifications $n=10$, or hotpot mutations alone $n=6$ ) in univariate (HR 2.59, $95 \% \mathrm{Cl} 1.21-5.55, p=0.015$ for mutation/amplification and $H R$ 1.37, 95\% Cl 0.44-4.31, $p=0.587$ for mutation alone) and multivariate ( $\mathrm{HR} 2.10,95 \% \mathrm{Cl} 0.94-4.67, \mathrm{p}=0.070$ for mutation/amplification and $\mathrm{HR}$ 1.37, $95 \% \mathrm{Cl} 0.41-4.53, \mathrm{p}=0.605$ for mutation alone) Cox proportional hazards regression models. Multivariate correction was undertaken for stage, sex, age at diagnosis of primary, non-synonymous mutation count as well for BRAF and NRAS mutation status. 